\title{
XLIV. A contribution to the theory of magnetic induction in iron and other metals.-Part II
}

\author{
John Buchanan D.Sc.
}

To cite this article: John Buchanan D.Sc. (1901) XLIV. A contribution to the theory of magnetic induction in iron and other metals._Part II, Philosophical Magazine Series 6, 2:11, 456-462, DOI: $10.1080 / 14786440109462711$

To link to this article: http://dx.doi.org/10.1080/14786440109462711

Published online: 08 Jun 2010.

Submit your article to this journal $₫$

ЏII Article views: 2

Q View related articles $\square$ 


\section{[ 456 ]}

XLIV. A Contribution to the Theory of Magnetic Induction in Iron and other Metals.-Part II. By JoHn Buchanan, D.Sc. (Lond.)*.

T $N$ the Phil. Mag. for March of this year there appeared a 1 paper of mine with the above title. It will be referred to below as Part I. We are now in a position to discuss in more detail the application of the general theory there given to some of Dr. Ewing's experimental results.

As in all such cases, the question resolves itself into the operation of determining constants-in other words, of finding the form of the graph which represents the initial conditions, such conditions being expressed by $x=0$ in (11) Part I. Work of this kind requires the expenditure of a good deal of time, so that $I$ am able to give here a first approximation only.

My objects in presenting these approximate results are to indicate the nature of the problem involved in the application of the general theory to the experimental facts, to suggest the form of the solution, and to compare the values of $x$ found here with those obtained in Part I. by an entirely different method.

The methods used and the results obtained will be found, I trust, of sufficient interest to justify their publication.

Curve for Annealed Iron ( $c f$. B. fig. 1, Part I.).

In the footnote, p. 336, Part I., I have given $x=1 \cdot 2$ for this curve, whilst the saturation intensity of magnetization (c) is given as 0.84 , that is $0.84 \times 1700=1428$ o.G S. units.

These results were obtained by the determination of the constants in the case where the specimen of iron was subjected to a continuous increase of magnetizing force.

I propose now to consider that curve of fig. 14, plate 59 of Dr. Ewing's paper in the Phil. Trans. pt. ii. 1885, which refers to the behaviour of the same specimen when the magnetizing force was varied cyclically.

This curve was subjected by me to harmonic analysis. The period was taken as $180 ; 90$ readings of I were taken off the curve at equal intervals of $\mathrm{H}$. By help of 4-figure mathe. matical tables I get as the harmonic constituents of the curve

$$
\begin{array}{r}
1521 \sin \left(\frac{2 \pi}{180} H-5^{\circ} \cdot 0\right) ; \\
468 \sin \left(\frac{6 \pi}{180} H-14^{\circ} \cdot 1\right) ; \\
273 \sin \left(\frac{10 \pi}{180} H-22^{\circ} \cdot 3\right) . \\
\text { * Communicated by the Authos. }
\end{array}
$$


The amplitudes of the octave and the third harmonic are inappreciable, as indeed the shape of the curve itself would suggest.

The presence of the epochs in the harmonic constituents is of course the indication of hysteresis and of residual magnetism in the material.

Equation (11) Part I. may be written in the form

$$
\mathrm{I}=\sum_{r=1}^{r=\infty} \mathrm{D}_{r} \epsilon^{-x} \sqrt{\bar{\pi}_{\overline{\mathrm{R}}}^{r}} \sin \left(\frac{2 \pi r}{\mathrm{R}} \mathrm{H}-x \sqrt{\overline{\mathrm{R}}} r+\theta_{r}\right), .
$$

where $\mathrm{D}_{r}, x$, and $\theta_{r}$ are constants whose values are to be determined.

For the curve in question it appears that $r$ assumes odd values only.

This last condition suggests a modification of fig. 5, Part I., as a possible form of the graph for $I_{x=0}$ which would apply to such an experimental curve as we have under examination.

A somewhat prolonged arithmetical study of the matter leads me to a general form of graph for $\mathrm{I}_{x=0}$ like that shown here in fig. 6 .

Fig. 6 .

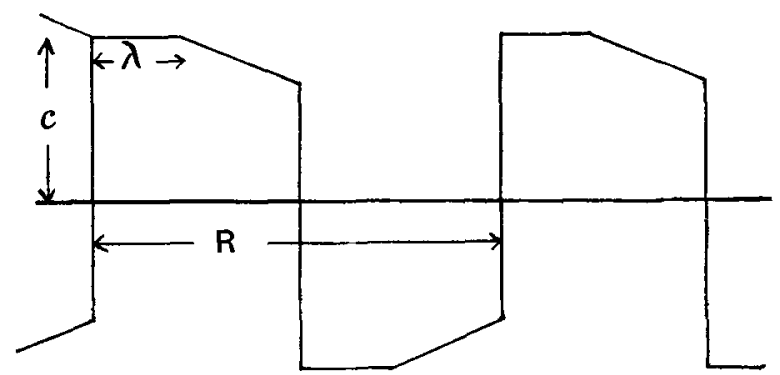

To find the equation to this graph, let

$$
\begin{aligned}
& \mathrm{I}_{x=0}=c \text { from } \mathrm{H}=0 \text { to } \mathrm{H}=\lambda ; \\
& \mathrm{I}_{x=0}=c-a(\mathrm{H}-\lambda) \text { from } \mathrm{H}=\lambda \text { to } \mathrm{H}=\frac{\mathrm{R}}{2} ; \\
& \mathrm{I}_{x=0}=-c \text { from } \mathrm{H}=\frac{\mathrm{R}}{2} \text { to } \mathrm{H}=\frac{\mathrm{R}}{2}+\lambda ; \\
& \mathrm{I}_{x=0}=-c+a\left(\mathrm{H}-\frac{\mathrm{R}}{2}-\lambda\right) \text { from } \mathrm{H}=\frac{\mathrm{R}}{2}+\lambda \text { to } \mathrm{H}=\mathbf{R} .
\end{aligned}
$$

By the application of Fourier's methods, we find as the 
equation required:

$$
\begin{aligned}
\mathrm{I}_{x=0} & =\frac{2 a \mathrm{R}}{\pi^{2}} \sum_{r=1}^{r=\infty} \frac{1}{r^{2}} \cos \frac{\pi r}{\mathrm{R}} \lambda \cos \frac{2 \pi r}{\mathrm{R}}\left(\mathrm{H}-\frac{\lambda}{2}\right) \\
& +\frac{4 c-a(\mathrm{R}-2 \lambda)}{\pi} \sum_{r=1}^{r=\infty} \frac{\sin \frac{2 \pi r}{\mathrm{R}} \mathrm{H}}{r} . \cdot
\end{aligned}
$$

in whtch $r$ has only odd values.

By comparison of the general terms of (15) and (16) with one another, we get

$$
\begin{aligned}
& \mathrm{D}_{r} \cos \theta_{r}=\frac{a \mathrm{R}}{\pi^{2} r^{2}} \sin \frac{2 \pi r}{\mathrm{R}} \lambda+\frac{4 c-a(\mathrm{R}-2 \lambda)}{\pi r} ; \\
& \mathrm{D}_{r} \sin \theta_{r}=\frac{2 a \mathrm{R}}{\pi^{2} r^{2}} \cos ^{2} \frac{\pi r}{\overline{\mathrm{R}}} \lambda . . . . . . . . .
\end{aligned}
$$

Thus $D_{r}$ and $\theta_{r}$ can be expressed in terms of the constants of the graph of $\mathrm{I}_{x=0}$.

Now, as Dr. Ewing has pointed out*, "the curves (of magnetization) ... appear in all cases to start off tangent to a line parallel to the axis on which $H$ is measured whenever the change of $\mathrm{H}$ is reversed in sign."

In the notation of (15) and (16) above, such reversal takes place when $\mathrm{H}=\frac{\mathrm{R}}{4}$. This would suggest that in (16) a value for $\lambda$ about $\frac{R}{4}$ should give an approximation to the truth.

Let us then take as an approximate value $\lambda=\frac{R}{4}$. By substitution in (17) we get

$$
\begin{aligned}
& \mathrm{D}_{r} \cos \theta_{r}=\frac{a \mathrm{R}}{\pi^{2} r^{2}} \sin \frac{\pi r}{2}+\frac{4 c-\frac{a \mathrm{R}}{2}}{\pi r} \\
& \mathrm{D}_{r} \sin \theta_{r}=\frac{a \mathrm{R}}{\pi^{2} r^{2}} . \quad . \quad . \quad . \quad . \quad . \quad . \\
& \therefore \tan \theta_{r}=\frac{a \mathrm{R}}{\pi r\left(4 c-\frac{a \mathrm{R}}{2}\right)+a \mathrm{R} \sin \frac{\pi r}{2}},
\end{aligned}
$$

* Phil. Trans. pt. ii. 1885, p. 533. 
or

$$
\tan \theta_{r}=\frac{2}{r Z-\pi r+2 \sin \frac{\pi r}{2}}, \quad . \quad .
$$

if we put

$$
\mathrm{Z}=\frac{8 \pi c}{a \mathrm{R}} \text {. }
$$

By help of (19) I drew curves on squared paper to show the connexion of $\tan \theta_{r}$ and $Z$ for the values $r=1, r=3, r=5$; the curves are of course hyperbolas. In this way was found a value of $\theta_{r}$ which, when substituted in (15), enables a value for $x$ to be determined such that the epochs obtained from the experimental curve of Dr. Ewing could be most nearly reproduced.

The nearest approximation I could get was

$$
\mathrm{Z}=22, \quad \theta_{1}=5^{\circ} \cdot 4, \quad \theta_{3}=2^{\circ} \cdot 1, \quad \theta_{5}=1^{\circ} \cdot 1, \quad \text { and } x=1 \cdot 33 \text {. }
$$

Thus, when these respective values of $x$ and $\theta_{r}$ are substituted in (15), we get as the harmonic constituents:-

$$
\begin{aligned}
& \mathrm{D}_{1} \epsilon^{-1 \cdot 33 \sqrt{\frac{\pi}{180}}} \sin \left(\frac{2 \pi}{180} \mathrm{H}-4^{\circ} \cdot 7\right) ; \\
& \mathrm{D}_{3} \epsilon^{-1 \cdot 33} \sqrt{\frac{\sqrt{3 \pi}}{180}} \sin \left(\frac{6 \pi}{180} \mathrm{H}-15^{\circ} \cdot 3\right) ; \\
& \mathrm{D}_{5} \epsilon^{-1 \cdot 33} \sqrt{\frac{5 \pi}{180}} \sin \left(\frac{10 \pi}{180} \mathrm{H}-21^{\circ} \cdot 4\right) .
\end{aligned}
$$

Moreover, if we substitute in (18) the value of $\mathrm{Z}$ just given, we can find $\mathrm{D}_{r}$. Thus

$$
\begin{aligned}
& \mathrm{D}_{1}=c \times 1.214 ; \\
& \mathrm{D}_{3}=c \times 0.4 ; \\
& \mathrm{D}_{5}=c \times 0.223 .
\end{aligned}
$$

Also, if we identify the calculated amplitudes of the harmonic constituents with those found from the experimental curve, we have

$$
\begin{aligned}
& \mathrm{D}_{1} \epsilon^{-1 \cdot 33} \sqrt{\frac{\pi}{180}}=1521 ; \\
& \mathrm{D}_{3} \epsilon^{-1 \cdot 33} \sqrt{\frac{3 \pi}{180}}=468 ; \\
& \mathrm{D}_{\tilde{\jmath}} \epsilon^{-1 \cdot 33} \sqrt{\frac{5 \pi}{180}}=273 .
\end{aligned}
$$


Or, using the values of $D_{1}, D_{3}$, and $D_{5}$ given immediately above,

Again,

$$
\begin{gathered}
c \times 1 \cdot 214 \times \mathrm{e}^{-1 \cdot 33} \sqrt{\frac{\pi}{180}}=1521, \\
\therefore c=1495 .
\end{gathered}
$$

$$
c \times 0^{*} 4 \times \epsilon^{-1 \cdot 33} \sqrt{\overline{3 \pi}}=468,
$$$$
\therefore c=1586 \text {. }
$$

Lastly,

$$
\begin{gathered}
c \times 0.223 \times \epsilon^{-1 \cdot 33} \sqrt{\frac{\overline{5 \pi}}{180}}=273, \\
\therefore c=1813 .
\end{gathered}
$$

The value of $c$ obtained from the different harmonic constituents should obviously be the same.

On reference to the first sentence in the discussion of this curve, it will be seen that the two values of $x$, viz. $1 \cdot 2$ and 1.33, are comparable with one another, whilst the value of $c$ there given is only strictly comparable with the first bere found, viz. 1428 with 1495 .

Curve for Hardened Iron ( $c f . \mathrm{C}$ and No. 3, fig. 1, Part I.).

On p. 336, Part I, 1 have given $x=4, c=1$, that is $c=1700$ C.G.S. units for curve No. 3, which coincides fairly well with Dr. Ewing's curve.

When the material is subjected to a cyclical variation of $\mathrm{H}$, harmonic analysis of Dr. Ewing's fig. 14, plate 59, loc. cit. for the specimen of iron which has been hardened by stretching, yields as the harmonic constituents

$$
\begin{gathered}
1235 \sin \left(\frac{2 \pi}{168} \mathrm{H}-8^{\circ .7}\right) \\
203 \sin \left(\frac{6 \pi}{168} \mathrm{H}-31^{\circ .8}\right) ; \\
78 \sin \left(\frac{10 \pi}{168} \mathrm{H}-55^{\circ} \cdot 2\right) .
\end{gathered}
$$

The amplitudes of the octave and of the third harmonic are again inappreciable.

With the same assumption regarding the value of $\lambda$ as was made above, I get

$$
\mathrm{Z}=7 \cdot 2, \theta_{1}=18^{\circ} \cdot 3, \theta_{3}=11^{\circ} \cdot 1, \theta_{5}=5^{\circ} \cdot 1 \text {, and } x=3 \cdot 4 \text {. }
$$


Magnetic Induction in Iron and other Metals.

By substitntion as before in (15) we get as the harmonic constituents :-

$$
\begin{aligned}
& D_{1} \epsilon^{-3 \cdot 4} \sqrt{16 \overline{3}}^{\bar{\pi}} \sin \left(\frac{2 \pi}{168} \mathrm{H}-8^{0.3} 3\right) ; \\
& \mathrm{D}_{3} \epsilon^{-3 \cdot 4} \sqrt{\frac{3 \pi}{168}} \sin \left(\frac{6 \pi}{168} \mathrm{H}-35^{\circ} \cdot 1\right) ; \\
& \mathrm{D}_{5} \epsilon^{-3 \cdot 4} \sqrt{\frac{5 \pi}{168}} \sin \left(\frac{10 \pi}{168} \mathrm{H}-54^{0.5}\right) .
\end{aligned}
$$

Using (18) we get

$$
\begin{aligned}
& \mathrm{D}_{1}=c \times 1 \cdot 132 ; \\
& \mathrm{D}_{3}=c \times 0.204 ; \\
& \mathrm{D}_{5}=c \times 0.144
\end{aligned}
$$

Or, by identification of amplitudes :-

$$
\begin{gathered}
c \times 1 \cdot 132 \times e^{-3 \cdot 1} \sqrt{\frac{\pi}{168}}=1235, \\
\therefore c=1736 .
\end{gathered}
$$

Again,

$$
\begin{gathered}
c \times 0 \cdot 204 \times \mathrm{e}^{-3 \cdot 4} \sqrt{\frac{3 \pi}{168}}=203, \\
\therefore c=2220 .
\end{gathered}
$$

Lastly,

$$
\begin{gathered}
c \times 0 \cdot 144 \times e^{-3 \cdot 4} \sqrt{\frac{5 \pi}{158}}=78, \\
\therefore c=1390 .
\end{gathered}
$$

As in the case of the previous curve, the two values of $x$, viz. 4.0 and $3 \cdot 4$, agree fairly well; of the values of $c$ only the first is practically identical with that given above, viz. 1736 with 1700 .

The results for thesetwo curves, approximate though they are, would appear to suggest the possibility of obtaining the value of $x$ and of the saturation intensity of magnetization for a material by help of the results of harmonic analysis of the curve $(I, H)$ when $H$ is varied cyclically.

That all the values of $c$ are of the same dimensions I consider to be sufficiently satisfactory, in view of the approximate nature of the harmonic analysis applied by mo to Dr. Ewing's curves, and in consideration also of the severity of the test which is applied when we extract from any curve by harmonic analysis such high period harmonics as the second and fourth. 
Recurring to the heat analogy, let us imagine the terminal portion of a very long conducting bar to be subjected to timeperiodic change of temperature. When the periodic changes bave become steady throughout the bar, let there be plotted the graphs of time-temperature for two sections of the bar whose distances from the end of the bar are, say, $u_{1}$ and $x_{2}$. In dealing numerically with the data obtained from the experiment, however, we can only use the value $\left(x_{2}-x_{1}\right)$, because practically the source of the changes of temperature operates not merely at the section $x=0$ but over a finite region in its neighbourhood. Conversely, if all the other data were known and we had to calculate the values of $x$ for the two sections of the bar, we could only expect to find an approximate agreement between the observed and the calculated values of $x$. The larger the value of $x$, the more close would be the approximation.

In the corresponding magnetic problem, my attention was directed to these considerations by the partial failure of a line of attack different from that of this paper, on the problem of determining constants.

How the question really stands is a matter for further research. Much light could be thrown on the whole subject by a systematic series of experiments on a material, such as an iron wire, by subjecting it to a cychcal variation of $\mathrm{H}$ of constant period whilst it is kept under a strain which is altered step by step. To make this clear, we might for example have

$H$ varying $0,+90,0,-90$, whilst the load on the wire is zero ;

$H$ varying $0,+90,0,-90$, whilst the load on the wire is 1 kilog.;

$\mathrm{H}$ varying $0,+90,0,-90$, whilst the load on the wire is 2 kilogs.; and so on.

A similar systematic series of experiments could be made with different temperatures, instead of with different loads, for the same period of $\mathrm{H}$.

I feel sure that the information obtained by the harmonic analysis of the results of such experiments would well repay the expenditure of the time and energy involved.

These suggestions I would take the liberty of recommending to the favourable consideration of the Advisory Committee of the National Physical Laboratory, in connexion with their proposal to experiment on the hysteresis of iron and its alloys.

Gordon's College, Aberdeen, August 1901. 